\title{
Chinese Children's Emotional Response Research Based on the Visual Stimulation of IAPS
}

\author{
Zhiyong Wang ${ }^{1}$, Zhenyu Shi ${ }^{2}$, Xirui Wang ${ }^{3}$, Jianqu Chen ${ }^{4}$, Honghai Liu ${ }^{1, *}$, Xiaodan Yu ${ }^{3, *}$ \\ ${ }^{1}$ School of Mechanical Engineering, Shanghai Jiao Tong University, Shanghai, China \\ ${ }^{2}$ Shanghai Agape Brain and AI Technology Company, Shanghai, China \\ ${ }^{3}$ Shanghai Children's Medical Center, Shanghai Jiao Tong University, Shanghai, China \\ ${ }^{4}$ School of Mechatronic Engineering and Automation, Shanghai University, Shanghai, China
}

\author{
Email address: \\ yzwang_sjtu@sjtu.edu.cn (Zhiyong Wang),zshi.harvard@yahoo.com (Zhenyu Shi), xirui_wang@foxmail.com (Xirui Wang), \\ chenjianqv@qq.com (Jianqu Chen), honghai.liu@sjtu.edu.cn (Honghai Liu),xdyu1108@163.com (Xiaodan Yu) \\ ${ }^{*}$ Corresponding author
}

\section{To cite this article:}

Zhiyong Wang, Zhenyu Shi, Xirui Wang, Jianqu Chen, Honghai Liu, Xiaodan Yu. Chinese Children's Emotional Response Research Based on the Visual Stimulation of IAPS. Science Discovery. Vol. 7, No. 5, 2019, pp. 266-271. doi: 10.11648/j.sd.20190705.12

Received: August 8, 2019; Accepted: September 24, 2019; Published: September 27, 2019

\begin{abstract}
It is a hot topic in related research that studying and evaluating of the emotion mental state and the emotional resonance through the emotional pictures which are used as a kind of visual stimulus. This paper mainly studies the induction of emotional valence and arousal of Chinese children based on the International Emotional Atlas System (IAPS) image, and develops a virtual environment interaction system for collecting multimodal information. A total of 198 children were invited to participate in the experimental test, of which the effective data was 178 groups. The article counts the response results of children's self-selected emotions' valence and arousal in different directions and degrees of image stimulation, and calculates the matching degree between picture stimulation and emotional expression under different error thresholds. The results showed that the proportion of emotional stimuli that can effectively induce children with the same valence was about $58.5 \%$, but different degrees of picture stimuli did not have emotional expression corresponding to arousal. Therefore, it is concluded that the first is to directly use the IAPS image system for the emotional induction of Chinese children needs improvement; the second is that this study shows multimodality based on other objective performances such as expressions, movements, eyes, and EEG activation. Analysis of children's emotions is very important.
\end{abstract}

Keywords: IAPS, Emotional Resonance, Virtual Environment Interaction, Visual Stimulation, Multisensing System

\section{基于IAPS视觉刺激的中国儿童情绪响应研究}

\author{
王志永 ${ }^{1}$, 石振宇 ${ }^{2}$, 王汐苾 ${ }^{3}$, 陈建驱 ${ }^{4}$, 刘洪海 ${ }^{1 *}$, 余晓丹 ${ }^{3^{*}}$ \\ ${ }^{1}$ 上海交通大学机械与动力工程学院, 上海, 中国 \\ ${ }^{2}$ 上海果效智能科技有限公司, 上海, 中国 \\ ${ }^{3}$ 上海交通大学医学院附属上海儿童医学中心发育行为儿科, 上海, 中国 \\ ${ }^{4}$ 上海大学机电工程与自动化学院, 上海, 中国
}

\section{邮箱}

yzwang_sjtu@sjtu.edu.cn（王志永）, zshi.harvard@yahoo.com（石振宇）, xirui_wang@foxmail.com（王汐芯）, chenjianqv@qq.com（陈建驱）, honghai.liu@sjtu.edu.cn（刘洪海）, xdyu1108@163.com（余晓丹） 
摘要: 通过情绪图谱诱发观察者情感共鸣, 研究其表现形式及作用效果是当前研究人员精神状态及情绪评估的一个热 门研究方向。本文主要研究基于国际情绪图谱系统（IAPS）图片对于中国儿童情绪效价及唤醒度的诱发情况, 并开发 了用于采集多模态信息的虚拟环境交互系统。共邀请了198名儿童参加实验测试, 其中有效数据为 178 组。文章统计了 在不同方向和程度的图片刺激作用下，儿童自选情绪的效价和唤醒度的响应结果，并计算了在不同误差阈值范围下， 图片刺激与情绪表达的匹配度。结果表明，不同效价的图片刺激能够有效诱发儿童产生相同效价的情绪共鸣的比例约 为 $58.5 \%$, 但不同程度的图片刺激未有对应唤醒度的情绪表达。因此, 本文得出结论, 一是直接将IAPS图片系统用于 中国儿童的情绪诱发需要改进; 二是本研究表明基于其他客观的表现, 比如表情, 动作, 眼神, 脑电激活等来多模态 分析儿童情绪具有非常重要的意义。

关键词：国际情绪图谱系统，情感共鸣，虚拟环境交互，视觉刺激，多传感系统

\section{1. 引言}

$0-5$ 岁的学前儿童中 $10 \%-14 \%$ 存在社交情绪行为的问 题[1]; 1/5的儿童有可达到诊断的某种精神障碍; 一半的 持续终生的精神障碍在 14 岁前起病, 24岁前3/4发病[2]。 精神障碍的总体上呈现出慢性化的特点, 及时发现和诊断 儿童的情绪异常具有重要意义。

儿童情绪异常患者普遍具有令自己心理不适的消 极情绪, 注意力缺失, 容易生气, 具有暴力倾向, 且通 常会表现出头部低陇、目光涣散、低度愉悦的苦笑、愁 眉、四监尬等表情, 且面部表现的持续时间及程度也与正 常人相差较远[3-4]。基于语言、面部表情、头部位置和 视线等方法开始逐渐开始用于精神异常的篮查和诊断 研究中。Mikhailova等[5]研究发现, 相对于健康人, 患 者识别面部表情的能力受损, 在辨别面部表情的测试中 会出现更多的错误; 而且在识别悲伤表情时反应时间较 长, 且表现出比较严重的消极倾向; Gollan等[6]研究发 现, 抑郁障碍患者减缓了处理悲伤一类的表情, 从而导 致其社会功能严重受损; Joormann等[7]研究发现抑郁障 碍患者对悲伤刺激表现出明显的注意偏向, 而健康对照 组被试则对高兴等正性刺激表现出明显偏向; Surguladze等[8]研究发现, 在社交场合中, 抑郁障碍、 焦虑障碍患者无法准确判断他人所表现出的面部表情 的微妙变化, 对表情的辨别精确度低, 无法精确判断诸 如快乐和愤怒等表情的微弱变化, 这可能就是构成人际 功能受损的原因。

获取及捕捉这些表现的前提是有一套能够很好诱发 儿童情绪共鸣的视觉诱导图片系统。国际情绪图片系统 (IAPS) 是美国情绪与注意研究中心情绪研究编制的标准 化图片系统[9-11], 已有研究表明在中国大学生群体中应 用IAPS, 在效价与唤醒度上的影响上均具有显著差异[9], 但直接将其应用于儿童情绪诱发实验中的效果还未有报 道。本研究重点研究基于IAPS图片系统, 用于中国儿童情 绪发育状态诱发的效价(Valence)及唤醒度(Arousal), 从而
为基于虚拟环境的儿童情绪笁查系统打下良好的前期研 究基础。

\section{2. 方法}

本文通过不同程度及类型的视觉刺激来诱发儿童情 感共鸣, 期间记录其头皮脑电（EEG）, 面部视频及儿童 在观看完一类图片后的自我认定情绪。提出了一种基于图 片刺激来对儿童情绪状态评估的预测方法。

\section{1. 实验系统}

本文开发了一个包括虚拟环境交互、脑电数据采集, 视频数据采集，语音数据采集的多模态融合采集系统。基 于Unity平台, 设计了一个基于虚拟环境交互的游戏, 将 用于儿童心理状态评估的问卷, 八类视觉刺激图片及其情 绪状态的认定, 以生动有趣的形式集成在一起, 用于采集 儿童的心理评估数据。如图1所示, 游戏中共有两个界面: 开始前准备界面以及测试界面。在开始前准备页面需要输 入儿童的名字缩写, 幼儿园名称缩写, 班级缩写以及完整 姓名并可进行测试麦克风语音测试, 并且在测试介绍框内, 会有本小游戏的规则说明。在测试见面, 在交互界面内, 会依次展示出不同类型的图片集, 供儿童观察, 观察完一 类图片后, 会进入一个情绪选择界面, 儿童根据自己当前 的心情, 选择不同的情绪。

头皮脑电作为一种生理神经信号, 一直被用于研究人 的情绪识别任务 [12-14]。本系统集成的脑电采集模块, 是 一款四通道小型化便携式的商业化脑电采集设备, 所得到 的脑电数据是利用脑电仪配套的软件进行采集及数据预 处理后得到的结果。视频数据的采集利用了笔记本电脑自 带的摄像头获取, 视频的帧率要求到达50PFS, 以使得能 够实时地捕捉到儿童在交互过程中的面部神态信息。系统 中的每个传感模块都配有时间标签, 可以用于数据的对齐 方便后续统一处理与分析。 


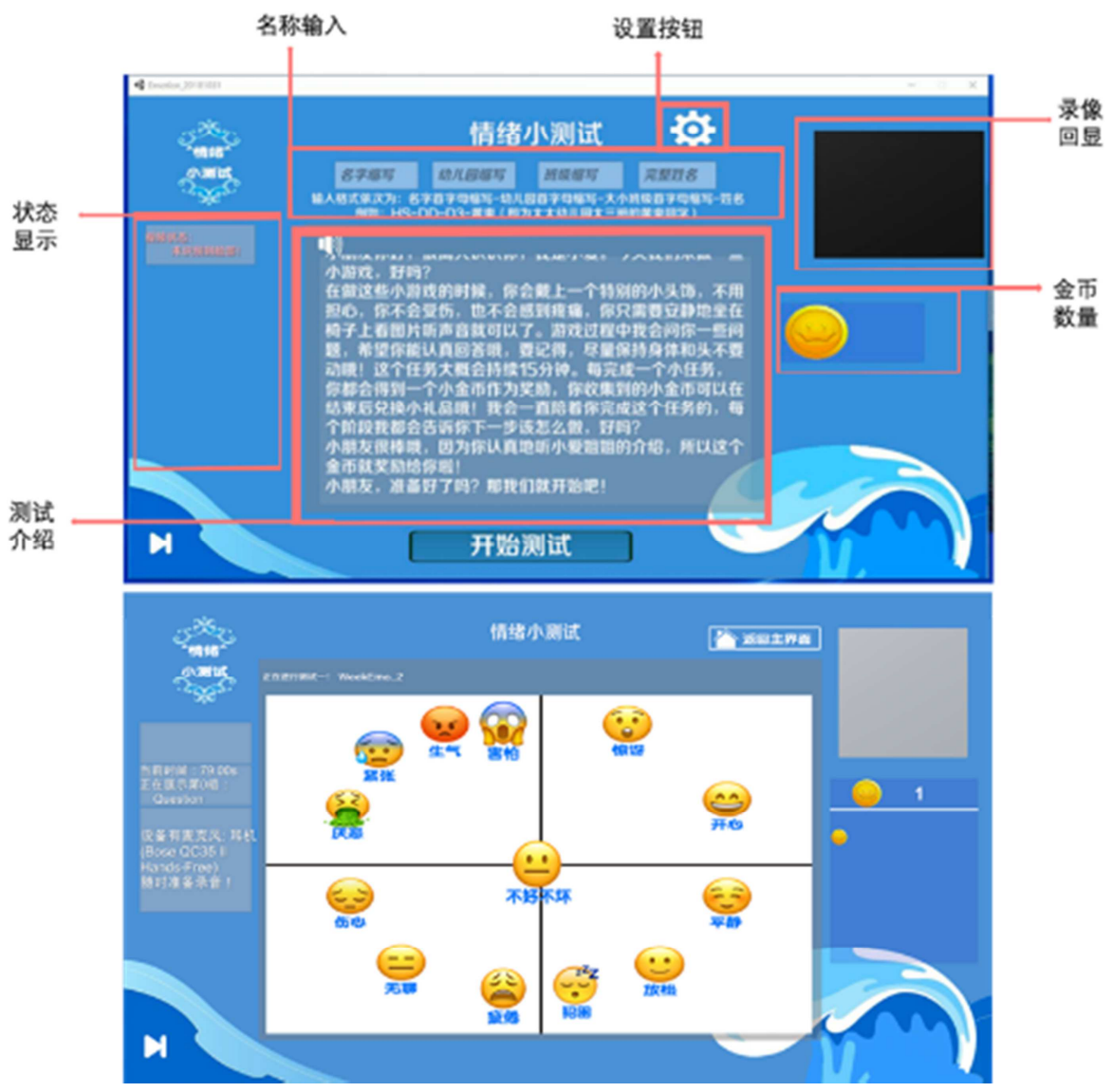

图1 虚拟交换环境界面。

\section{2. 实验流程}

本实验, 共分为三个环节, 实验测试前准备环节, 测 试环节一及测试环节二三个部分，如图2所示。首先测试 前准备环节, 在老师和儿童自己的描述下, 填好自己信息, 并进行语音, 脑电及视频模块的测试, 保证各模块可以正 常运行, 可以清晰的采集到需要的信息。然后, 经过简单 的游戏规则介绍后, 就进入测试环节一。测试环节一主要 测试儿童一周的情绪状态，共包含四个部分。第一，系统 会通过语音提示, 询问儿童一周内的心情怎么样; 第二, 儿童在听完提示后, 会被引导选择一个与自己情绪相近的 表情; 第三，系统会再次询问儿童一周的心情，并希望儿 童可以自述; 第四, 儿童自述一周的心情。语音检测到说 话结束后，在每次儿童有反馈时，会奖励儿童一个小金币 用于激励儿童继续进行游戏。测试环节二主要是通过图片 刺激的模式来激发小孩子的情感共鸣。国际情绪图片系统 (International Affective Picture System, IAPS) 是情绪研究 的常用工具。IAPS共有八类图片, 其中包扩从最负面到最 正面的八个分级 (NHH, NHL, NLH, HLL, PLL, PLH, PHL,PHH）, 每类图片4张, 共32张图片, 示例图片如图3 所示。测试环节二一共八组实验, 每组播放一类图片, 每 张图片放映 10 秒, 在 40 秒后, 会再次进入表情选择界面和 自述心情界面。在测试的过程中，儿童的脑电信号及视频 被完整的保存下来以供后续数据分析。

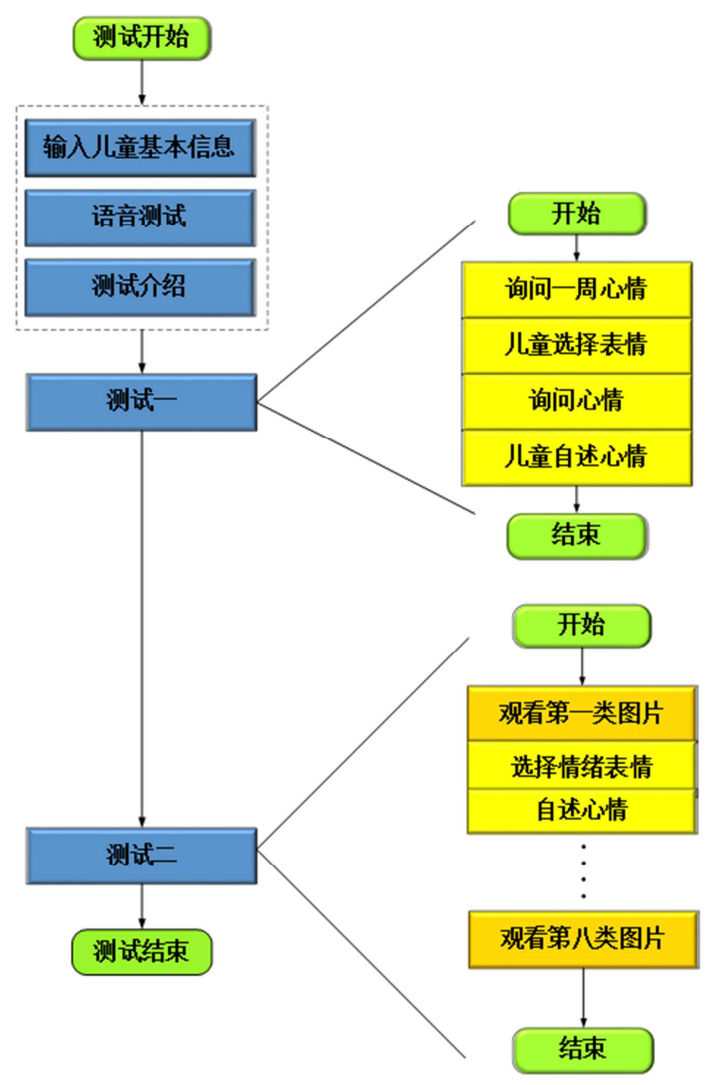

图2 测试流程图。 


\section{3. 数据处理与分析}

论本文主要研究基于IAPS系统的图片系统诱发正常 小孩情绪共鸣情况, 故仅分析儿童在观察图片后的情绪选 择的情况。

将十三种情绪按照效价和唤醒度量化设定坐标值, 具 体如图4所示。并将八类图片根据不同的刺激方向及程度 赋予数字描述, 具体表 1 所示。

表1 量化IAPS图谱刺激方向及程度。

\begin{tabular}{llllllll}
\hline 图片 & 刺激 & 图片 & 刺激 & 图片 & 刺激 & 图片 & 刺激 \\
类型 & 程度 & 类型 & 程度 & 类型 & 程度 & 类型 & 程度 \\
\hline NHH & -2 & NHL & -1.5 & NLH & -1 & NLL & -0.5 \\
PLL & 0.5 & PLH & 1 & PHL & 1.5 & PHH & 2 \\
\hline
\end{tabular}

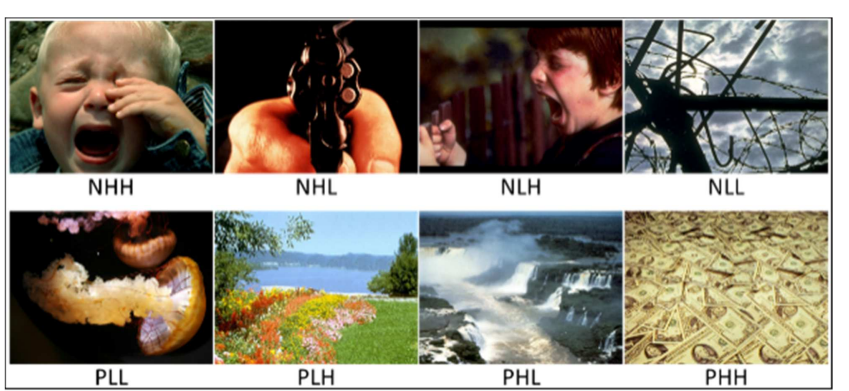

图3 IAPS示例图片。

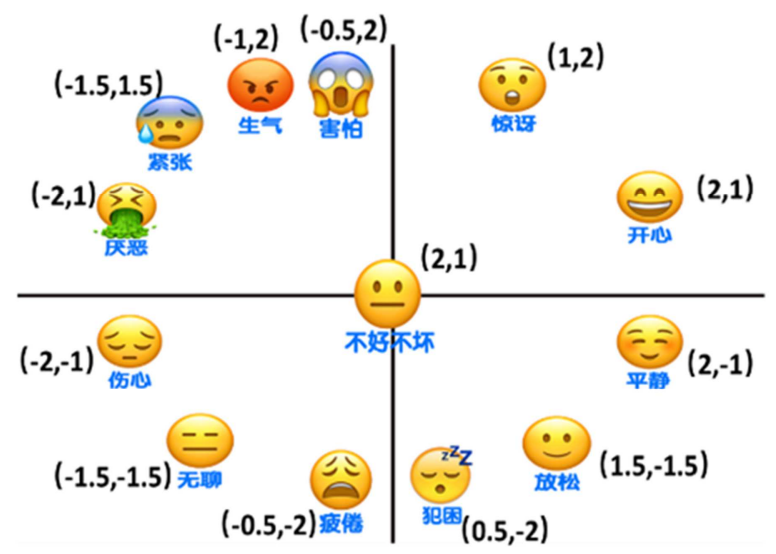

图4 坐标化心情选择图。

本研究共采集到了198名儿童的数据, 其中一些儿童 由于个人原因未能完成全部的实验, 故剔除该部分数据, 剩余有效数据为 178 条。本研究从两个角度来研究儿童对 于图片刺激的研究, 第一是从图片刺激的正面情绪和负面 情绪来分析, 即研究当刺激图片是正面积极的内容时, 诱 发出儿童表现正面情绪的概率, 以及当刺激图片是负面消 极内容时, 诱发出儿童表现负面情绪的概率。第二是从儿 童自选情绪与界定的图片刺激方向与程度的距离, 即在不 同误差阈值下, 计算自选的情绪与图片刺激方向与程度的 匹配关系。

针对研究一, 在x坐标维度上, 能够很好的反应出儿 童情绪的积极程度, 即 $\mathrm{X}$ 越往右, 情绪越积极越正面。故 统计样本中在不同刺激方向 (-2 2) 下, 儿童自选情绪横 坐标的正负情况, 从而反应儿童在观看不同刺激方向后的
情绪波动情况。在 $\mathrm{y}$ 坐标维度上, 能够很好的反应儿童情 绪的唤醒度, 即坐标值越往上, 情绪越激动, 唤醒度越高。 故统计在不同刺激程度 $(0.5 \sim 2,-0.5 \sim-2)$ 下, 儿童自选 情绪纵坐标的正负情况, 从而反映儿童在观察图片后情绪 的唤醒度状态及程度。

针对研究二, 则是在给定的误差阈值 $\mathrm{e}$ ( $\mathrm{e}$ 取值为 $1,1.5$, 2）内, 计算儿童自选情绪状态与图片刺激的方向及程度的 匹配度。如果自选情绪坐标值与情绪图片标定值得绝对数 值小于 $\mathrm{e}$,则认为在误差 $\mathrm{e}$ 允许的情况下, 图片刺激诱发了儿 童的情绪共鸣, 反之, 则表示图片刺激未能引起儿童共鸣。

\section{3. 结果}

针对研究一, 在X维度, 统计了178位儿童, 在观察了 八类图片后的情绪选择状态对应的横坐标的正负情况。统 计结果如图5所示, 结果表明, 对于正面图片的刺激, 有 约 $75.7 \%$ 的儿童的情绪表现为积极, 而约 $24.3 \%$ 的儿童, 选 择了偏消极情绪。对于负面图片的刺激, 有约 $41.4 \%$ 的儿 童的情绪表现为消极, 而约 $58.6 \%$ 的儿童仍然选择了较为 积极的情绪状态。整体来看, 通过图片的积极程度来诱发 儿童产生共鸣的成功率约为 $58.5 \%$ 。

在Y维度, 将四个刺激程度 $( \pm 0.5, \pm 1, \pm 1.5, \pm 2)$ 简化 为两个层次 (高刺激, 低刺激)。统计了儿童在观察了八 类图片后的情绪选择状态对应的纵坐标的正负情况。结果 如表2 所示。结果显示, 在高程度刺激和低程度刺激图片 对于儿童唤醒度的影响, 并没有显著的差异。

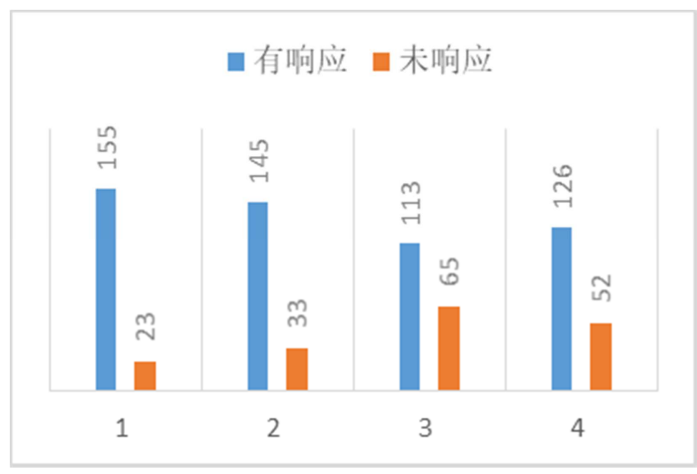

（1）正面刺激情绪相应图

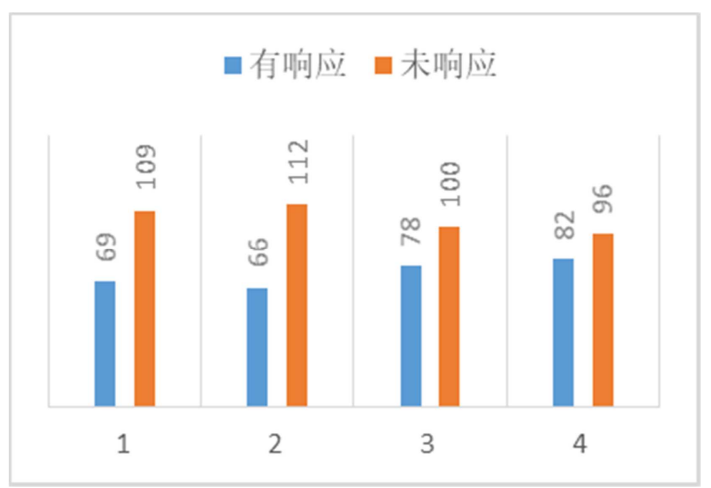

（2）负面刺激情绪相应图

图5 图片刺激诱发情绪效价响应图。 
表2

不同刺激程度下情绪唤醒度。

\begin{tabular}{lll}
\hline \multicolumn{1}{c}{ 刺激程度 } & 高刺激 & 低刺激 \\
\hline 兴奋程度 & $488(68.5 \%)$ & $470(66 \%)$ \\
\hline 兴奋度高 & $224(31.5 \%)$ & $242(34 \%)$ \\
兴奋度低 & & \\
\hline
\end{tabular}

针对研究二, 统计了在不同误差阈值的范围下，效价和 唤醒度两个维度上, 图片刺激与情绪响应的匹配度, 结果如 图6和图7所示。结果表明, 在误差阈值e设定为 1.5 及 2 时, 大 部分的图片类型对于情绪的诱发是有效的, 但误差阈值e设 定为 1 时, 即在相对精准的情绪刺激上, 数据波动很大, 尤 其对于唤醒度的影响还出现了与刺激程度完全相反的情况, 及在图片刺激程度较高时，儿童情绪相应相对低迷。

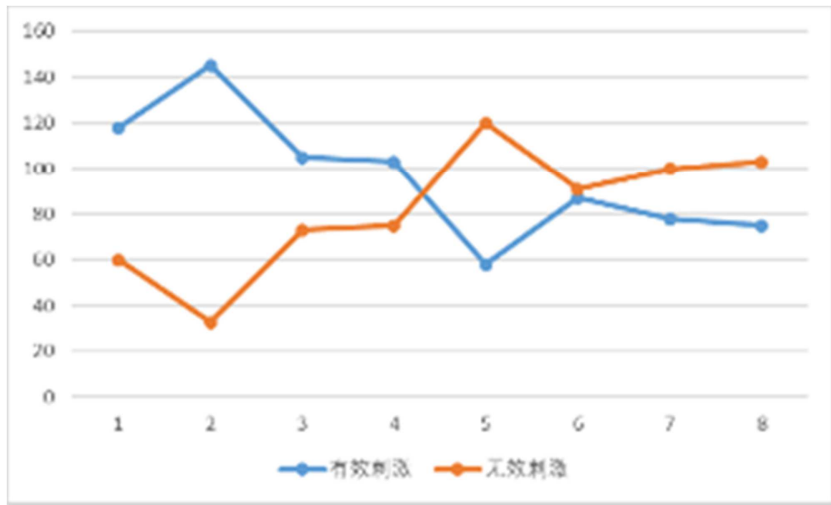

(1) $\mathrm{e}=1$

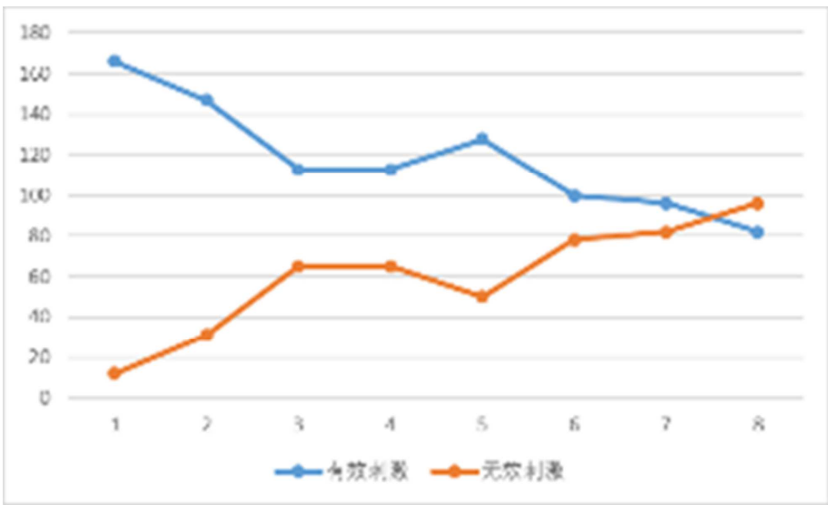

(2) $\mathrm{e}=1.5$

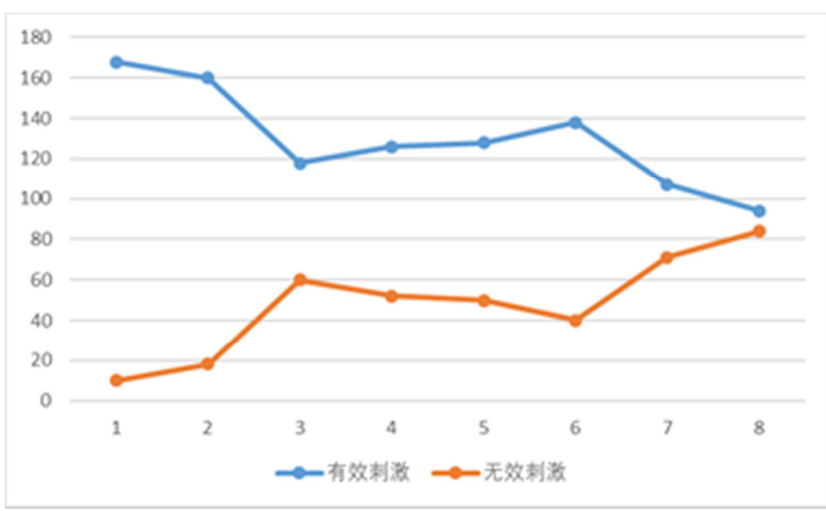

(3) $\mathrm{e}=2$

图6 不同误差阈值下图片刺激对情绪效价的影响。

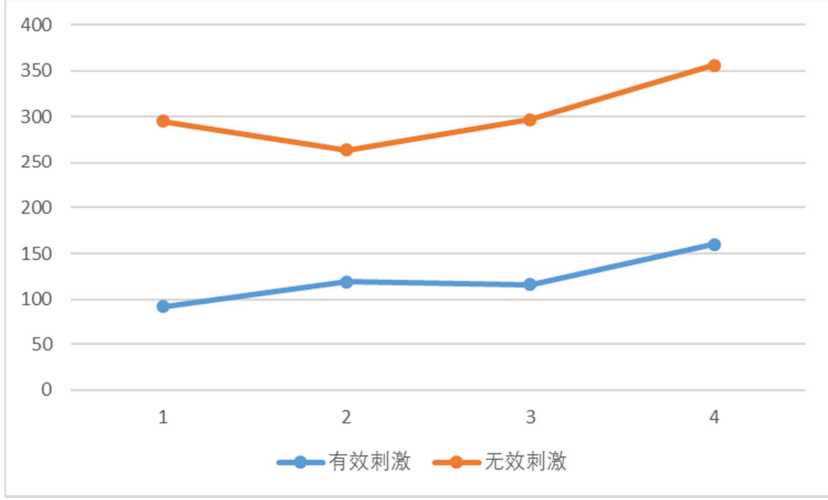

(1) $\mathrm{e}=1$

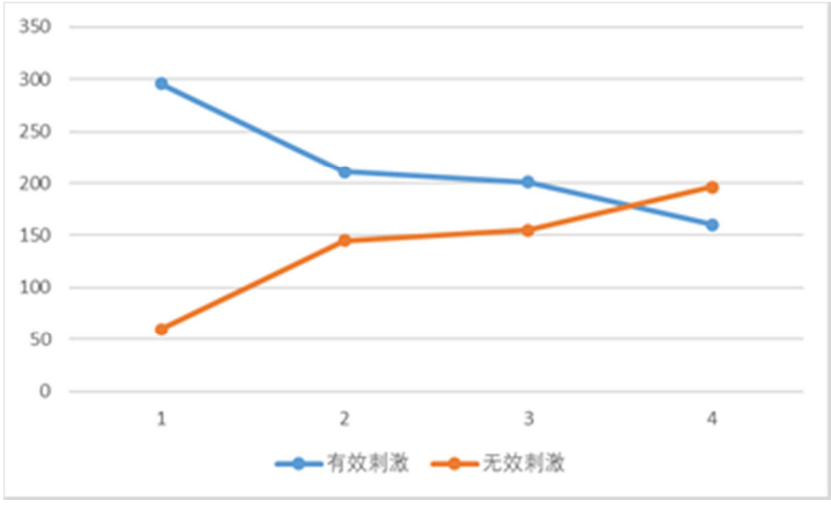

(2) $\mathrm{e}=1.5$

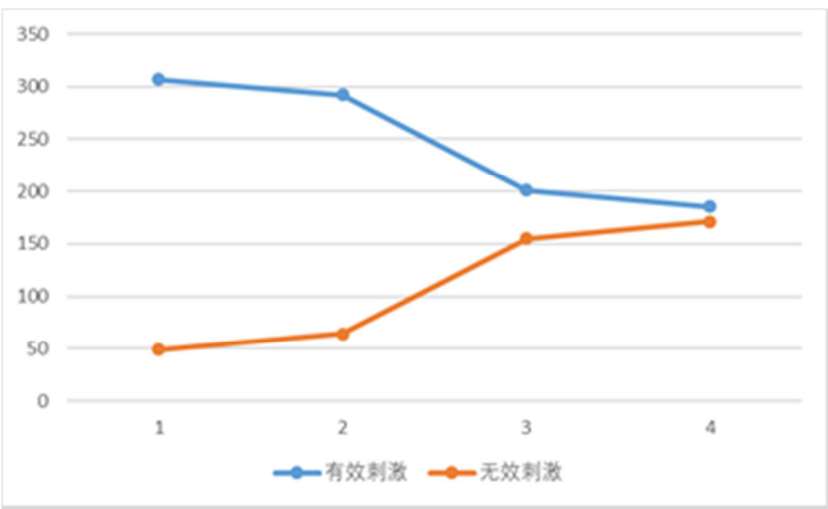

(3) $\mathrm{e}=2$

图7 不同误差阈值下图片刺激对情绪唤醒度的影响。

\section{4. 结论}

本文重点分析了基于国际情绪图片系统（IAPS）的儿 童情绪诱发状态在效价和唤醒度方向的影响, 实验结果表 明, 不同效价的图片刺激能够相对有效的诱发儿童产生相 同效价的情绪共鸣, 但不同刺激程度的图片刺激未有对应 唤醒度的情绪表达。原因可能有以下几点: 一、学龄前 儿童脑发育尚处于情绪识别和分化和自我觉察初期阶段, 对于标准化处理后的图片（如平和、冲突、血腥等），尚 不能产生完全的同理化的情绪体验, 他们往往会表现出好 奇或无所谓的状态, 尚不能完全准确低选择表示真实的情 绪状态图示[15]。这种结果也和基础、临床研究一致, 即 
儿童的情绪问题, 多以外化后行为的方式来呈现; 而随着 年龄的增加, 会逐渐以抑郁焦虑等内化的方式呈现[16]。 因此, 基于其他客观的表现, 比如表情, 动作, 眼神, 脑 电激活等来分析其情绪状态具有非常重要的研究意义。二、 直接将IAPS图片系统本身是基于美国人情绪状态而研发 的图片系统, 由于中美文化差异及生活环境的不同, 儿童 对于图片内容的理解本身就欠缺, 未能准确捕捉到图片内 容表达的信息, 故而未能引起情绪上的共鸣。

本文提出的基于虚拟环境交互的多模态感知系统具 有很好的应用前景, 在后续研究中, 将结合儿童的面部状 态, 脑电激活程度来研究儿童的情绪状态, 并结合临床情 绪状态异常儿童的数据来分析其情绪表达的差异性。

\section{致谢}

本文是国家重点研究计划重点专项 （2016YFC1305204），上海市浦东新区科学技术委员会 项目（PKJ2017-Y05）, 上海交通大学医工交叉重点项目

（YG2017ZD15），上海大学大学生联合大作业项目《基 于脑机互动和AI心理对话的抑郁焦虑情绪改善系统》的阶 段性成果之一。

\section{参考文献}

[1] Brauner, C.B. and C.B. Stephens, Estimating the prevalence of early childhood serious emotional/behavioral disorders: challenges and recommendations. Public Health Rep, 2006. 121 (3): p. 303-10.

[2] Egger, H.L. and A. Angold, Common emotional and behavioral disorders in preschool children: presentation, nosology, and epidemiology. J Child Psychol Psychiatry, 2006. 47 (3-4): p. 313-37.

[3] E. W. McGinnis et al., "Movements Indicate Threat Response Phases in Children at Risk for Anxiety," in IEEE Journal of Biomedical and Health Informatics, vol. 21, no. 5, pp. 1460-1465, Sept. 2017.

[4] C. S and V. K, "An Approach to Measure and Improve the Cognitive Capability of ADHD Affected Children Through EEG Signals," 2018 IEEE 18th International Conference on Advanced Learning Technologies (ICALT), Mumbai, 2018, pp. 314-318.
[5] Mikhailova ES, Vladimirova TV, Iznak AF. Abnormal recognition of facial expression of emotions in depressed patients with major depression disorder and schizotypal personality disorder. Biol Psychiatry. 1996, 40 (8): 697-705.

[6] Gollan JK, Pane HT, McCloskey MS. Identifying differences in biased affective information processing in major depression. Psychiatry Res. 2008, 159 (1-2): 18-24.

[7] Joormann J, Gotlib IH. Selective attention to emotional faces following recovery from depression. J Abnorm Psychol. 2007, 116 (1): 80-85.

[8] Surguladze SA, Young AW, Senior C. Recognition accuracy and response bias to happy and sad facial expressions in patients with major depression. Neuropsychology. 2004, 18 (2): 212-218.

[9] Freudenberger HJ. Staff burnout. Journal of Social Issues, 1973, 30 (1): 159-165.

[10] Maslach C, Schaufeli WB, Leiter MP. Job Burnout. Annual Reviews of Psychology, 2001, 52: 397-422.

[11] 刘潇楠, 许咱翔, 周仁来. 国际情绪图片系统的本土化研 究: 在中国大学生群体中的评定 $[\mathrm{J}]$. 中国临床心理学杂志, 2009 (6):687-689。

[12] B. Xing et al., "Exploiting EEG Signals and Audiovisual Feature Fusion for Video Emotion Recognition," in IEEE Access, vol. 7, pp. 59844-59861, 2019.

[13] N. Akalin and H. Köse, "Emotion recognition in valence-arousal scale by using physiological signals," 2018 26th Signal Processing and Communications Applications Conference (SIU), Izmir, 2018, pp. 1-4.

[14] L. Faes, A. Greco, A. Lanata, R. Barbieri, E. P. Scilingo and G. Valenza, "Causal brain-heart information transfer during visual emotional elicitation in healthy subjects: Preliminary evaluations and future perspectives," 2017 39th Annual International Conference of the IEEE Engineering in Medicine and Biology Society (EMBC), Seogwipo, 2017, pp. 1559-1562.

[15] 梁宗保,张光珍,陈会昌,张萍. 学前儿童情绪理解的发展及 其与父母元情绪理念的关系 [J]. 心理发展与教 育,2011(3):233-240。

[16] Erol N, Simsek Z, Oner O, Munir K. Behavioral and emotional problems among Turkish children at ages 2 to 3 years. J Am Acad Child Adolesc Psychiatry. 2005 Jan; 44 (1): 80-7. 